\title{
Venous circulation and the invisible hand
}

\author{
Robert H. Thiele, MD
}

Received: 22 November 2017 / Accepted: 22 November 2017/Published online: 27 December 2017

(C) Canadian Anesthesiologists' Society 2017

Adam Smith, the Scottish economist, philosopher, and writer, used the phrase "invisible hand" to describe the ability of some unknowable force to match the supply of goods with demand in market economies, ultimately achieving a state of equilibrium defined by a price that is agreeable to both parties in an economic transaction. ${ }^{1}$ While the exact meaning of the phrase is still debated almost 250 years later, it is clear that Smith's “invisible hand" concept was intended to simplify extraordinarily complex phenomena-the economic decisions of a society of self-interested individuals - and suggested that rule sets, which could not be understood or perceived by individuals, could produce a measurable effect on society.

Smith's framework for breaking down complex systems into understandable bits and pieces and simplified rules is prevalent throughout science, including biology. We are taught, for instance, that cardiac output (CO) is equal to the mean arterial pressure (MAP) divided by systemic vascular resistance (SVR). However, MAP and SVR are abstractions and do not actually exist-i.e., blood pressure is a continuous, pulsatile variable dependent on time and location, and SVR as a measurable function of afterload is a gross simplification that completely neglects the pulsatile nature of the cardiovascular system. Indeed, impedance, which takes into account the periodic nature of flow, is a much more appropriate measure of afterload, but can be difficult to understand. ${ }^{2}$

It should therefore come as no surprise that these "simplified" models—-be they in biologic or even health

\section{R. H. Thiele, MD ( $\square)$}

Department of Anesthesiology, University of Virginia School of Medicine, P.O. Box 800710, Charlottesville, VA 22908-0710, USA

e-mail: rht7w@virginia.edu economic systems—-sometimes fail. For example, in my own Virginian community, the price of Affordable Care Act-compliant health insurance for a family of four recently increased by $300 \%$ to over $\$ 36,000 /$ year. ${ }^{3}$ Families who can afford insurance are purchasing it, and those who cannot are going without. This binary reality is hardly the smooth continuum of prices that Smith would have predicted for such a fundamentally important highfrequency economic transaction. It turns out that the "invisible hand" is actually quite complicated and can be distorted by regulation, subsidies, tax policies, and the interaction between public and private institutions.

Similarly, in biology, control of the cardiovascular system is extraordinarily complex. Yes, an "invisible hand" makes sure that left and right ventricular $\mathrm{CO}$ are nearly equal and that venous return and $\mathrm{CO}$ are matched. But armed with the overly simplistic knowledge that $\mathrm{CO}=$ MAP/SVR, clinicians may be tempted to think that interventions that increase MAP are likely to increase CO. This thinking neglects SVR, which for the majority of patients is unmeasurable and is a result of what has been called "tangible bias...our tendency to favor what we can see and understand over what we cannot". 4,5

In this issue of the Journal, Bigatello and Gelman describe the most important and most neglected component of the "invisible hand" that maintains cardiovascular stability and function-the systemic venous vasculature ${ }^{6}$ -important, because the venous system can profoundly affect cardiovascular function, as Guyton demonstrated in $1959 ;{ }^{7}$ neglected, because most clinicians do not routinely measure venous pressures, and those who do often do so incorrectly. ${ }^{8}$ Bigatello and Gelman's manuscript is not for the faint of heart (or mind), but it is a must read for anyone who wishes to more fully understand physiology so that clinical outcomes can be optimized. 
Guyton's own early work, discussed extensively by Bigatello and Gelman, illustrates nicely how complex venous physiology can be. In one early study, Guyton produced venous function curves in instrumented animals at varying doses of epinephrine. He found that increasing doses of epinephrine led to increased mean circulatory pressure and venous return. ${ }^{9}$ Notably, measured venous resistance was unaffected by epinephrine doses ranging from $0-3.5 \mu \mathrm{g} \cdot \mathrm{kg}^{-1} \cdot \mathrm{min}^{-1}$. Unfortunately, the use of a drug with both alpha- and beta-adrenergic activity limits the physiologic information that could be gleaned from this study. In a more informative study in which arterial and venous resistances were independently modified, Guyton found that $\mathrm{CO}$ was highly sensitive to small changes in venous resistance and relatively insensitive to changes in arterial resistance. ${ }^{7}$ This study had significant implications for clinical practice as it implied that a relatively healthy heart could easily respond to changes in afterload (it is, after all, a big bag of muscles), but could do nothing in the face of decreased venous return. And, because the system venous system depends on relatively low pressure gradients to drive blood into the right atrium, small changes in resistance, whether due to venoconstriction or obstruction, can produce a disproportionate impact.

Also critical to understanding cardiovascular physiology is the concept of stressed (under pressure) and unstressed (not under pressure) volume, a phenomenon unique to the systemic venous system and one nicely elucidated by Bigatello and Gelman. In their paper, they describe how in a severely hypovolemic patient, administered fluid is likely to be first deposited in unstressed venous compartments, offering no detectable hemodynamic benefit. In this instance, a patient will behave as a "non-responder" in goal-directed fluid therapy terminology, and clinicians may be inclined to discontinue what might otherwise be lifesaving therapy-only a strong and nuanced understanding of venous physiology, combined with situational awareness, can prevent this potential catastrophe. Complicating matters further, commonly used vasoactive agents may affect the ratio of stressed to unstressed volume, which will of course impact venous return and CO. In particular, alpha agonists such as phenylephrine and dopamine have been shown to decrease systemic venous compliance in humans. ${ }^{10,11}$

As goal-directed therapy and tighter control over intravascular volume becomes increasingly prevalent in perioperative and critical care settings, understanding the interaction among the systemic venous vasculature, left and right ventricles, and intravascular volume will become increasingly important. Bigatello and Gelman's manuscript is a great place to start.

\section{La circulation veineuse et $l a$ main invisible}

Pour Adam Smith, économiste, philosophe et écrivain écossais, le concept de «main invisible » décrit la capacité d'une force inconnue à faire correspondre, dans les économies de marché, l'approvisionnement des biens à la demande, pour atteindre en bout de ligne un état d'équilibre, défini par un prix jugé acceptable par les deux parties d'une transaction économique. ${ }^{1}$ Alors que la signification exacte de ce terme fait encore l'objet de débats près de 250 ans plus tard, il ne fait aucun doute que le concept de «main invisible» de Smith avait pour intention première de simplifier des phénomènes extraordinairement complexes, soit les décisions économiques d'une société composée d'individus intéressés, et sous-entendait que des ensembles de règles, qui ne pouvaient être compris ou perçus par l'individu, pouvaient avoir un effet mesurable sur la société.

Le procédé que Smith utilise pour décomposer des systèmes complexes en éléments compréhensibles et en règles simplifiées est prévalent dans toutes les sciences, $\mathrm{y}$ compris la biologie. Par exemple, on nous apprend que le débit cardiaque (DC) est égal à la tension artérielle moyenne (TAM) multipliée par la résistance vasculaire systémique (RVS). Toutefois, la TAM et la RVS sont des concepts abstraits qui n'existent pas réellement - en d'autres termes, la tension artérielle est une variable continue et pulsatile dépendant du moment et de l'emplacement; quant à la RVS, en tant que fonction mesurable de la post-charge, elle est une simplification grossière qui ne tient aucun compte de la nature pulsatile du système cardiovasculaire. En réalité l'impédance, qui prend en considération la nature périodique du débit, est une mesure bien plus adaptée de la post-charge, mais elle peut être difficile à comprendre. ${ }^{2}$

Ainsi, nous ne devrions pas être surpris si ces modèles « simplifiés »- qu'il s'agisse de systèmes biologiques ou de systèmes économiques de la santé - soient parfois erronés. Par exemple, dans ma propre collectivité, en Virginie, le prix d'une assurance santé conforme au Affordable Care Act, pour une famille de quatre personnes, a récemment augmenté de $300 \%$ pour atteindre plus de 36000 US\$/année. ${ }^{3}$ Les familles qui peuvent se payer une assurance l'achètent, et ceux qui ne peuvent pas, s'en passent. Cette réalité binaire est bien loin du continuum sans heurt des prix que Smith aurait envisagé pour une assurance maladie, une transaction économique très fréquente et d'une importance si fondamentale. Il semble que la «main invisible» soit en fait plutôt complexe et puisse être faussée tant par des lois, que des 
subsides, des politiques de taxes ou encore par l'interaction entre les institutions publiques et privées.

De la même manière, en biologie, le contrôle du système cardiovasculaire est extraordinairement complexe. Oui, une « main invisible » s'assure que le DC gauche et droite soient presque égaux et que le retour veineux corresponde au DC. Cependant, armés de la connaissance trop simpliste que le DC $=$ TAM $x$ RVS, les cliniciens pourraient être tentés de prendre pour acquis que les interventions augmentant la TAM augmenteront vraisemblablement le DC. Ce type de raisonnement ne tient pas compte de la RVS qui, chez la majorité des patients, ne peut être mesurée : c'est le résultat de ce qu'on a nommé « un biais tangible... soit notre propension à préférer ce que nous pouvons voir et comprendre plutôt que ce qui nous échappe »., ${ }^{4,5}$

Dans ce numéro du Journal, Bigatello et Gelman décrivent la composante la plus importante et la plus négligée de la « main invisible » qui maintient la stabilité et la fonction cardiovasculaires: la vasculature veineuse systémique. ${ }^{6}$ Cette composante est à la fois importante, parce que le système veineux peut affecter profondément la fonction cardiovasculaire, comme Guyton l'a démontré en $1959,{ }^{7}$ et négligée, parce que la plupart des cliniciens ne mesurent pas systématiquement les pressions veineuses, et ceux qui la mesurent le font souvent de façon incorrecte. ${ }^{8}$ Le manuscrit de Bigatello et Gelman ne s'adresse pas aux âmes (ou aux esprits) sensibles, mais c'est une lecture incontournable pour toute personne qui souhaite mieux comprendre la physiologie afin d'optimiser les pronostics cliniques.

Les premiers travaux de Guyton, que Bigatello et Gelman discutent en profondeur, illustrent bien la complexité de la physiologie veineuse. Dans une de ses premières études, Guyton a créé des courbes de la fonction veineuse d'animaux de laboratoire recevant diverses doses d'épinéphrine. Il a découvert que des doses croissantes d'épinéphrine entraînaient une augmentation de la pression circulatoire moyenne et du retour veineux. ${ }^{9}$ Fait notable, la résistance veineuse mesurée n'a pas été affectée par des doses d'épinéphrine allant de 0-3,5 $\mu \mathrm{g} \cdot \mathrm{kg}^{-1} \cdot \mathrm{min}^{-1}$. Malheureusement, en raison de l'activité combinée alpha- et bêta-adrénergique de ce médicament, les renseignements physiologiques qu' on aurait pu glaner de cette étude sont limités. Dans une étude plus informative dans laquelle les résistances artérielle et veineuse ont été modifiées de manière indépendante, Guyton a découvert que le DC était très sensible à de petits changements de la résistance veineuse, alors qu'il était relativement insensible aux changements de résistance artérielle. ${ }^{7}$ Cette étude a d'importantes implications pour la pratique clinique, en ce qu'elle sous-tend qu'un cœur en relativement bonne santé pourrait facilement réagir à des changements de post-charge (le cœur n'étant, après tout, qu'un gros sac de muscles), mais ne pouvait rien faire lorsque confronté à une réduction du retour veineux. Qui plus est, étant donné que le système veineux systémique dépend de gradients de pression relativement bas pour pousser le sang dans l'oreillette droite, de petits changements de résistance, qu'ils soient dus à une vasoconstriction veineuse ou à une obstruction, peuvent avoir un impact disproportionné.

Un autre concept essentiel à la compréhension de la physiologie cardiovasculaire est celui de volume contraint (sous pression) ou non contraint (pas sous pression), un phénomène rencontré uniquement avec le système veineux systémique et brillamment élucidé par Bigatello et Gelman. Dans leur article, ils décrivent la façon dont, chez un patient gravement hypovolémique, les fluides administrés se déposeront probablement d'abord dans les compartiments veineux non contraints, ne procurant donc aucun bienfait hémodynamique détectable. Dans cet exemple, un patient se comportera comme un «non-répondant » dans le jargon de la thérapie liquidienne ciblée, et les cliniciens pourraient envisager d'interrompre ce qui constituerait autrement un traitement salvateur; seule une compréhension solide et nuancée de la physiologie veineuse, combinée à une bonne conscience situationnelle, pourrait prévenir cette catastrophe potentielle. Pour compliquer les choses, les agents vasoactifs fréquemment utilisés pourraient affecter le rapport entre volume contraint et volume non contraint, ce qui aura évidemment un impact sur le retour veineux et le DC. En particulier, il a été démontré que les agents alphaagonistes tels que la phényléphrine et la dopamine réduisaient la compliance veineuse systémique chez l'humain. $^{10,11}$

La prévalence de la thérapie ciblée et d'un contrôle plus rigoureux du volume intravasculaire augmente dans les contextes périopératoires et de soins critiques; dès lors, il sera de plus en plus important de comprendre l'interaction entre la vasculature veineuse systémique, les ventricules gauche et droit, et le volume intravasculaire. Pour ce faire, le manuscrit de Bigatello et Gelman est un excellent point de départ.

Conflicts of interest None declared.

Editorial responsibility This submission was handled by Dr. Hilary P. Grocott, Editor-in-Chief, Canadian Journal of Anesthesia.

\section{Conflit d'intérêt Aucun.}

Responsabilité éditoriale Cet article a été traité par Dr Hilary P. Grocott, rédacteur en chef, Journal canadien d'anesthésie.

\section{References}

1. Thornton M. Cantillon and the invisible hand. Q J Austrian Econ 2009; 12: 27-46.

2. O'Rourke $M F$. The arterial pulse in health and disease. Am Heart J 1971; 82: 687-702. 
3. Itkowitz C. Where are the most expensive ACA plans in America? Charlottesville. Washington Post. November 16, 2017. Available from URL: https://www.washingtonpost.com/local/where-are-themost-expensive-aca-plans-in-america-charlottesville/2017/11/16/ e1b352d8-ca27-11e7-aa96-54417592cf72_story.html?utm_term=. b876ac90646b (accessed November 2017).

4. Thiele RH, Nemergut EC, Lynch C 3rd. The physiologic implications of isolated alphal adrenergic stimulation. Anesth Analg 2011; 113: 284-96.

5. Magder S. Phenylephrine and tangible bias. Anesth Analg 2011; 113: 211-3.

6. Gelman S, Bigatello L. Physiologic basis for goal-directed hemodynamic and fluid therapy: the pivotal role of the venous circulation. Can J Anesth 2018; 65. https://doi.org/10.1007/ s12630-017-1045-3.

7. Guyton AC, Abernathy B, Langston JB, Kaufmann BN, Fairchild $H M$. Relative importance of venous and arterial resistances in controlling venous return and cardiac output. Am J Physiol 1959; 196: 1008-14.

8. Figg KK, Nemergut EC. Error in central venous pressure measurement. Anesth Analg 2009; 108: 1209-11.

9. Guyton AC, Lindsey AW, Abernathy B, Langston JB. Mechanism of the increased venous return and cardiac output caused by epinephrine. Am J Physiol 1958; 192: 126-30.

10. Marino RJ, Romacnoli A, Keats AS. Selective venoconstriction by dopamine in comparison with isoproterenol and phenylephrine. Anesthesiology 1975; 43: 570-2.

11. Eichler HG, Ford GA, Blaschke TF, Swislocki A, Hoffman BB. Responsiveness of superficial hand veins to phenylephrine in essential hypertension. Alpha adrenergic blockade during prazosin therapy. J Clin Invest 1989; 83: 108-12. 